\title{
On the Predictability of Rainfall in Kerala An Application of ABF Neural Network
}

\author{
Ninan Sajeeth Philip and K. Babu Joseph \\ Cochin University of Science and Technology, Kochi-682022, Kerala State, India. \\ nspp@iucaa.ernet.in
}

\begin{abstract}
Rainfall in Kerala State, the southern part of Indian Peninsula in particular is caused by the two monsoons and the two cyclones every year. In general, climate and rainfall are highly nonlinear phenomena in nature giving rise to what is known as the 'butterfly effect'. We however attempt to train an ABF neural network on the time series rainfall data and show for the first time that in spite of the fluctuations resulting from the nonlinearity in the system, the trends in the rainfall pattern in this corner of the globe have remained unaffected over the past 87 years from 1893 to 1980 . We also successfully filter out the chaotic part of the system and illustrate that its effects are marginal over long term predictions.
\end{abstract}

\section{Introduction}

Although technology has taken us a long way towards better living standards, we still have a significant dependence on nature. Rain is one of nature's greatest gifts and in third world countries like India, the entire agriculture depends upon rain. It is thus a major concern to identify any trends for rainfall to deviate from its periodicity, which would disrupt the economy of the country. This fear has been aggravated due to the threat by the global warming and green house effect. The present study has a soothing effect since it concludes that in spite of short term fluctuations, the general pattern of rainfall in Kerala has not undergone major deviations from its pattern in the past.

The geographical configuration of India with the three oceans, namely Indian Ocean, Bay of Bengal and the Arabian sea bordering the peninsula gives her a climate system with two monsoon seasons and two cyclones inter-spersed with hot and cold weather seasons. The parameters that are required to predict the rainfall are enormously complex and subtle so that the uncertainty in a prediction using all these parameters is enormous even for a short period. The period over which a prediction may be made is generally termed the event horizon and in best results, this is not more than a week's time. Thus it is generally said that the fluttering wings of a butterfly at one corner of the globe may cause it to produce a tornado at another place geographically far away. This phenomenon is known as the butterfly effect.

The objective of this study is to find out how well the periodicity in these patterns may be understood using a neural network so that long term predictions 
can be made. This would help one to anticipate with some degree of confidence the general pattern of rainfall for the coming years. To evaluate the performance of the network, we train it on the rainfall data corresponding to a certain period in the past and cross validate the prediction made by the network over some other period. A difference diagram ${ }^{1}$ is plotted to estimate the extent of deviation between the predicted and actual rainfall. However, in some cases, the cyclone may be either delayed or rushed along due to some hidden perturbations on the system, for example an increase in solar activity. (See figure 2.) These effects would appear as spikes in the difference diagram. The information from the difference diagram is insufficient to identify the exact source of such spike formation. It might have resulted from slight perturbations from unknown sources or could be due to an inaccurate modeling of the system using the neural network. We thus use a standard procedure in statistical mechanics that can quantitatively estimate the fluctuations [1]. This is to estimate the difference in the Fourier Power Spectra (FPS) of the predicted and actual sequences. In the FPS, the power corresponding to each frequency interval, referred to as the spectral density gives a quantitative estimate of the deviations of the model from reality. Rapid variations would contribute to high frequency terms and slowly varying quantities would correspond to low frequency terms in the power spectra.

The degree of information that may be extracted from the FPS is of great significance. If the model agrees with reality, the difference of the power spectra ( hereafter referred to as the residual FPS ) should enclose minimum power in the entire frequency range. An exact model would produce no difference and thus no residual FPS pattern. If there are some prominent frequency components in the residue, that could indicate two possibilities; either the network has failed to comprehend the periodicity, or that there is a new trend in the real world which did not exist in the past. One can test whether the same pattern exists in the residual FPS produced on the training set and confirm whether it is a new trend or is the drawback of the model.

A random fluctuation would be indicated in the residual FPS by amplitudes at all frequency values as in the case of 'white' noise spectra. Here again, how much power is enclosed within the FPS gives a quantitative estimate of the perturbations. A low amplitude fluctuation can happen due to so many reasons. But its effect on the overall predictability of the system would be minimal. If however, the residual FPS encloses a substantial power from the actual rainfall spectrum, the fluctuations could be catastrophic.

In this study, the results indicate that the perturbations produced by the environment on the rainfall pattern of Kerala state in India is minimal and that there is no evidence to envisage a significant deviation from the rainfall patterns prevailing here.

The general outline of the paper is as follows. In section 2 we present a brief outline of the Adaptive Basis Function Neural Network (ABFNN), a variant of

\footnotetext{
${ }^{1}$ This is the diagram showing the deviation of the predicted sequence from the actual rainfall pattern.
} 
the popular Back-Propagation algorithm. In section 3, the experimental set up is explained followed by the results and concluding remarks in section 4

\section{Adaptive Basis Function Neural Networks}

It was shown in 2] that a variant of the Back-Propagation algorithm (backprop) known as the Adaptive Basis Function Neural Network performs better than the standard backprop networks in complex problems. The ABFNN works on the principle that the neural network always attempts to map the target space in terms of its basis functions or node functions. In standard backprop networks, this function is a fixed sigmoid function that can map between zero and plus one or between minus one and plus one the input applied to it from minus infinity to plus infinity. It has many attractive properties that make the backprop an efficient tool in a wide variety of applications. However serious studies conducted on the backprop algorithm have shown that in spite of its widespread acceptance, it systematically outperforms other classification procedures only when the targeted space has a sigmoidal shape 3. This implies that one should choose a basis function such that the network may represent the target space as a nested sum of products of the input parameters in terms of the basis function. The ABFNN thus starts with the standard sigmoid basis function and alters its nonlinearity by an algorithm similar to the weight update algorithm used in backprop.

Instead of the standard sigmoid function, ABFNN opts for a variable sigmoid function defined as

$$
O_{f}=\frac{a+\tanh (x)}{1+a}
$$

Here $a$ is a control parameter that is initially set to unity and is modified along with the connection weights along the negative gradient of the error function. It is claimed in 22 that such a modification could improve the speed and accuracy with which the network could approximate the target space.

The error function is computed as:

$$
E=\sum_{k} \frac{\left(O_{k}-O_{k}^{*}\right)^{2}}{2}
$$

with $O_{k}$ representing the network output and $O_{k}^{*}$ representing the target output value.

With the introduction of the control parameter, the learning algorithm may be summarized by the following update rules. It is assumed that each node, $k$, has an independent node function represented by $a_{k}$. For the output layer nodes, the updating is done by means of the equation:

$$
\Delta a_{k}=-\beta\left(O_{k}-O_{k}^{*}\right) \frac{1-O_{k}}{1+a_{k}}
$$

where $\beta$ is a constant which is identical to the learning parameter in the weight update procedure used by backprop. 
For the first hidden layer nodes, the updating is done in accordance with the equation:

$$
\Delta a_{k-1}=-\sum_{i} w_{i j} \beta\left(O_{k}-O_{k}^{*}\right)\left(1-O_{k}\right)\left[\left(1+a_{k}\right)+O_{k}\left(1+a_{k}\right)\right] \frac{\partial O_{k}}{\partial O_{k-1}}
$$

Here $w_{i j}$ is the connection weight for the propagation of the output from node $i$ to node $j$ in the subsequent layer in the network.

The introduction of the control parameter results in a slight modification to the weight update rule $\left(\Delta w_{i j}\right)$ in the computation of the second partial derivative term $\frac{\partial O_{j}}{\partial I_{j}}$ in:

as:

$$
\Delta w_{i j}=-\beta \frac{d E}{d w_{i j}}=-\beta \frac{\partial E}{\partial O_{j}} \frac{\partial O_{j}}{\partial I_{j}} \frac{\partial I_{j}}{\partial w_{i j}}
$$

$$
\frac{\partial O_{j}}{\partial I_{j}}=\left[\left(1-a_{j}\right)+\left(1+a_{j}\right) O_{j}\right]\left(1-O_{j}\right)
$$

The algorithm does not impose any limiting values for the parameter $a$ and it is assumed that care is taken to avoid division by zero.

\section{Experimental Setup}

In pace with the global interest in climatology, there has been a rapid updating of resources in India also to access and process climatological database. There are various data acquisition centers in the country that record daily rainfall along with other measures such as sea surface pressure, temperature etc. that are of interest to climatological processing. These centers are also associated to the World Meteorological Organization (WMO). The database used for this study was provided by the Department of Atmospheric Sciences of Cochin University of Science and Technology, a leading partner in the nation wide climatological study centers.

The database consists data corresponding to the total rainfall in each month of Trivandrum city in Kerala, situated at latitude-longitude pairs $\left(8^{\circ} 29^{\prime} \mathrm{N}\right.$ $76^{\circ} 57^{\prime} \mathrm{E}$ ). Although the rainfall data from 1842 were recorded, there were many missing values in between that we had to restrict to periods for which a continuous time series was available. This was obtained for the period from 1893 to 1980.

For training our network, the monthly rainfall database from 1893 to 1933 was used. This corresponds to the first 480 data samples in the database. Since rainfall has an yearly periodicity, we started with a network having 12 input nodes each with the corresponding month's total rainfall as input. It was observed that the network accuracy increases systematically as we increased the number of input nodes from 12 to 48 covering the data of 4 years. The training RMS error in this case went as low as 0.0001 in about 3000 iterations. Any increase in input nodes resulted in poorer representations. However, the performance of the network on the remaining test data was found to be poor due to 
over fitting in the training data. This happened because of the large number of free parameters in the network compared to the size of the training set. Further experimentation showed that it is not necessary to input the data of all the twelve months of the previous 4 years, but, four sets of 3 months data centered over the predicted month of the fifth year in the 4 previous years will give good generalization properties. We thus finalized our network with 12 input nodes each for the 3 months input data over 4 years, 7 hidden nodes and one output node. In this way, based on the information from the four previous years around the targeted month, the network is to predict the amount of rain to be expected in the same month of the fifth year.

The training was carried out until the RMS error stabilized to around 0.085 over the training data(40 years from 1893-1933). The ABFNN converged to this value in around 350 iterations starting from random weight values.

After training, testing was carried out independently on the remaing test data set. For generating the difference diagram, the data corresponding to the entire 87 years of rainfall in Trivandrum city was presented to the network. The difference of the output produced by the network to the actual rainfall in each month was computed and plotted. This diagram enables one to make an easy estimate of the error levels in both the training and test periods. For a quantitative estimate of generalization error on the independent test set, the power spectrum of the output produced by the network was subtracted from the power spectra of the actual rainfall data in those months. This residual spectra was then compared with the spectral differences between the actual patterns in the training and test periods. In a dynamic system, there will always be some residual spectra. But if the network could generalize well and capture the dynamics in the training data, the output produced by it with the input from the test period will be more identical to the actual pattern. This means, the residual spectra will contain lesser residual power. If this power is less than the power contained in the difference between the spectra of the training and test periods of the actual rainfall patterns, it indicates that the deviations are not due to any new phenomenon, but are due to the trends that existed from the training period itself. Now the concern is the power content of the residual spectra. The lower the power enclosed in this residual spectra, the better is the learning and the generalization. The advantage of the scheme is that it provides a platform for comparing the performance of various networks with ease and allows one to visualize the spectral components that have actually deviated.

\section{Results and Discussion}

In figure 1 we show the difference pattern produced by the network over the entire 87 year period on the Trivandrum database. The training period is also shown. It may be noted that the deviations of the predicted signal from the actual in the entire dataset falls within the same magnitude range. The root mean square error on the independent test dataset was found to be around 0.09 . The two positive spikes visible in the plot corresponding to the two instances 


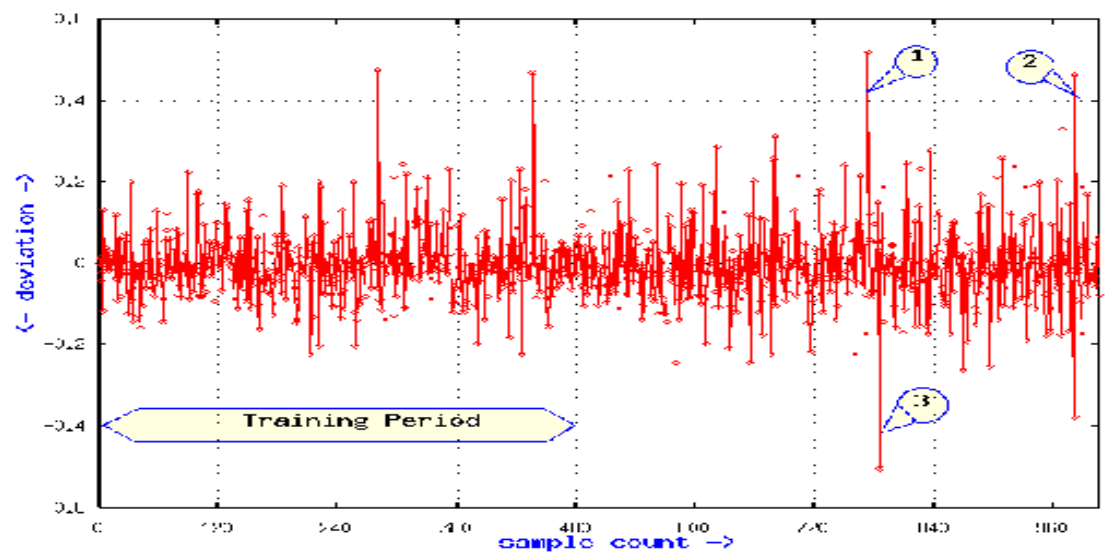

Fig. 1. The deviation of the predicted sequence from the actual time series data is shown. It may be noted that the deviations in the training period and the rest of the series are comparable in magnitude. The larger spikes are mainly due to the delay in the actual commencement of the monsoon or cyclone. Label 1 indicates an example where the monsoon extended into the following month giving more rainfall than anticipated. Label 2 in the figure represent an example where the monsoon was actually delayed by one month than was anticipated thus producing two spikes, one negative and the other positive. Label 3 corresponds to a negative spike caused by the change in trend from the predicted increase in rain in the following year.

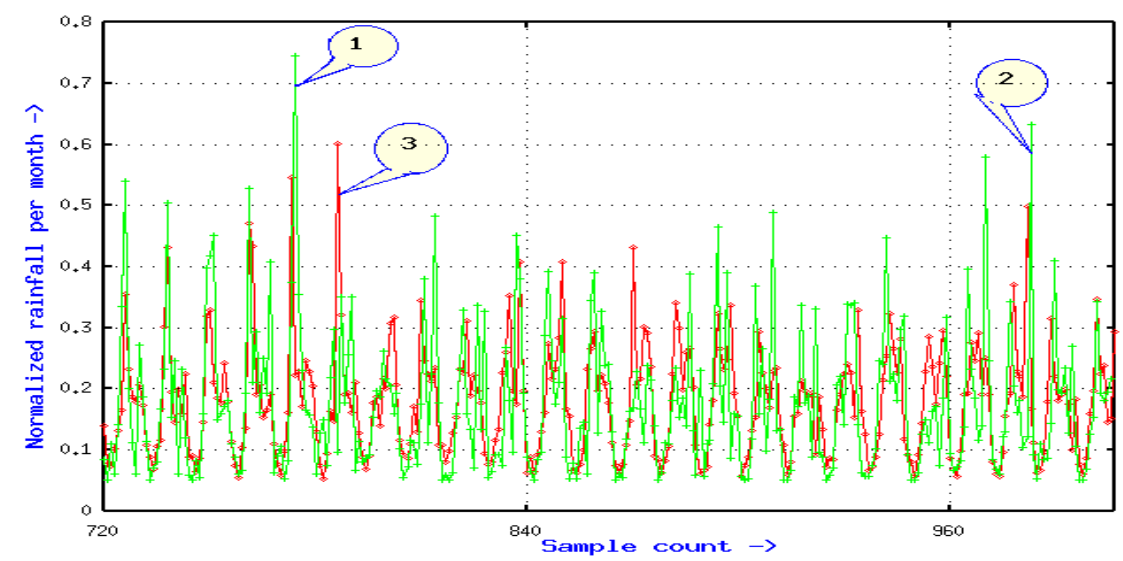

Fig. 2. An overlap of the predicted and the actual time series data in the region corresponding to the spikes in figure 1 is shown. The labels indicate the reason for the spikes seen in figure 1 . 
where the rainfall was delayed with reference to the month for which it was predicted are shown in figure 2

The cause for the negative spike in figure 1 is also seen in figure 2 as due to the deviation of the time series from the predicted upward trend. Factors such as the El-Nino southern oscillations (ENSO) resulting from the pressure oscillations between the tropical Indian Ocean and the tropical Pacific Ocean and their quasi periodic oscillations are observed to be the major cause of these deviations in the rainfall patterns 4.

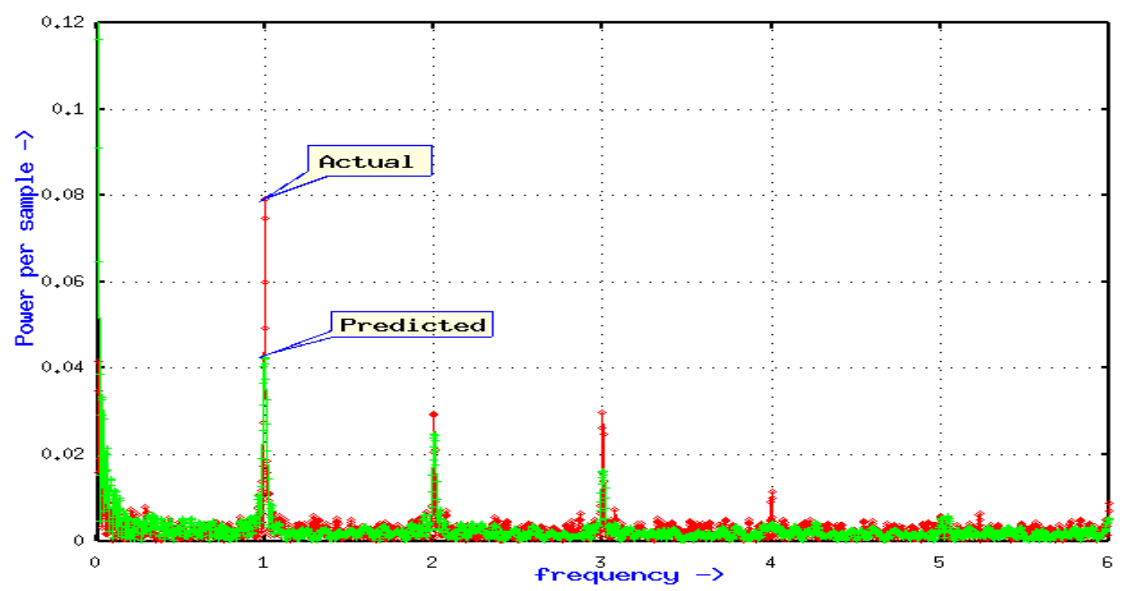

Fig. 3. The Fourier power spectra corresponding to the actual and the predicted rainfall for the entire 87 years. The sampling frequency does not have much significance here since the sample values represent the total rain received in each month. It was taken to be twelve to associate itself with the 12 months of the year. Since FPS is symmetric over half the sampling frequency, we show here only values from 0 to 6 .

To obtain a quantitative appreciation of the learning algorithm, we resort to the spectroscopic analysis of the predicted and actual time series. The average power per sample distributed in the two sequences are shown in figure 3, It is seen that the two spectra compare very well. We show the deviation of the FPS of the predicted rainfall data from the actual in the test period in figure 4(top). The second figure in figure 4 shows the deviation of the FPS of the actual rainfall data in the test period from that corresponding to the training period. This residual spectra indicates the deviation in periodicities and quantity of rainfall during the test period as compared to that in the training period. But it is seen that in the top figure most of the new frequency components visible in the second figure are missing. From the scaling property of Fourier spectra, one knows that power deviations in the spectra indicates deviations in the quanta of rainfall. Such deviations are indicators of the error associated with the prediction and are caused by the nonlinearity in the global factors that affect rainfall. New trends, or deviations of the existing rainfall pattern are identified by new frequency 

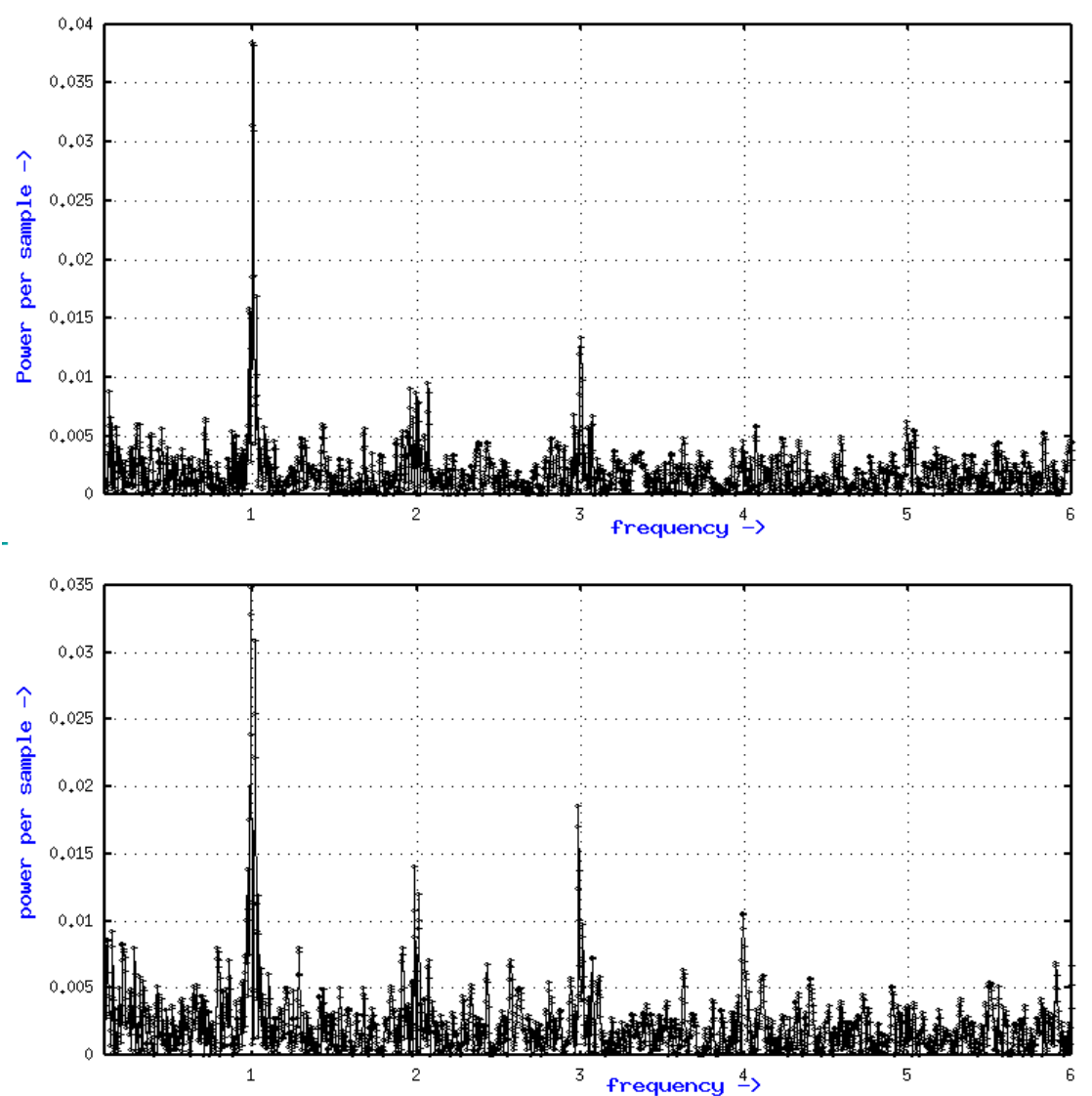

Fig. 4. The residual FPS corresponding to the predicted and actual rainfall in the years following the training period is shown on top. The spectral difference from a Fourier analysis perspective (the difference of the FPS in the training period and the period corresponding to the top figure) is presented in the bottom figure. Note that the spectral differences are minimum in the top figure. This is to say that the deviations observed in the actual spectra in the bottom figure followed from the trends that existed in the training period. 
components and not by the amplitudes of the component frequencies. The fact that there are no major new frequency components in the top figure suggests that the network could capture those trends seen in the second figure from the training data itself and that the rainfall trend in the test period has not changed considerably from the training period. In other words, it is to be concluded that other than minor fluctuations, the power factor in the residual FPS is insignificant to expect any major deviations in the rainfall pattern prevailing in the country.

The above discussion also shows that the ABF neural network is a better tool than the popular Fourier analysis methods for predicting long term rainfall behavior. The learning ability of the network can give a concise picture of the actual system. Fourier spectra is a transformation from time domain to frequency domain on the assumption that the time domain sequence is stationary. It thus fails to represent the dynamics of the system which is inherent in most natural phenomena and rainfall in particular.

A comparative study of the network with standard back-propagation network(BPNN) was also done. It was found that two hidden layers with 12 nodes each were required for obtaining a comparable convergence in the BPNN. The training and test errors after 1000 iterations were found to be respectively 0.954 and 0.948. It is to be noted that even with about three times more hidden nodes, the BPNN is having a higher RMS error after training it for about two times as much iterations used by ABFN. This clearly indicates that updating the basis function makes sense in complex problems.

Acknowledgment. The authors wish to express their sincere thanks to Professor K. Mohankumar of the Department of Atmospheric Sciences of the Cochin University of Science and Technology for providing us with the rainfall database. The first author would like to thank Dr. Moncy V John of the Department of Physics, Kozhencherri St. Thomas college for long late night discussions.

\section{References}

1. E. S. R. Gopal, Statistical Mechanics and Properties of Matter Theory and Applications, Ellis Horwood, 1974.

2. N. S. Philip and K. B. Joseph, Adaptive Basis Function for Artificial Neural Networks, (in press) Neurocomputing Journal, also available at http://www.geocities.com/sajithphilip/research.htm

3. M. A. Kraaijveld Small sample behaviour of multi-layer feed forward network classifiers: Theoretical and Practical Aspects (Delft University Press, 1993).

4. A. Chowdhury and S. V Mhasawade, Variations in meteorological floods during summer monsoon over India, Mausam, 42, 2, 167-170, 1991. 